\title{
Determination of Five Sulfonamides in Water by High Performance Liquid Chromatography Combined with lonic Liquid Liquid-liquid Microextraction
}

\author{
Gao Yuling ${ }^{1,2}$, Liang Ting ${ }^{3}$, Sun Peng ${ }^{1,2}$, Guo Yongxia ${ }^{1, *}$ \\ ${ }^{1}$ College of Agriculture, Heilongjiang Bayi Agricultural University, Daqing, China \\ ${ }^{2}$ Agricultural Products and Procesed Products Supervision and Testing Center, Ministry of Agriculture, Daqing, China \\ ${ }^{3}$ Agricultural and rural area Bureau of Daiyue District, Tai'an City, Taian, China
}

Email address:

guoyongxia@byau.edu.cn (Guo Yongxia)

*Corresponding author

\section{To cite this article:}

Gao Yuling, Liang Ting, Sun Peng, Guo Yongxia. Determination of Five Sulfonamides in Water by High Performance Liquid Chromatography Combined with Ionic Liquid Liquid-liquid Microextraction. Science Journal of Analytical Chemistry. Vol. 8, No. 3, 2020 , pp. 89-93. doi: $10.11648 /$ j.ajac.20200803.14

Received: June 4, 2020; Accepted: June 24, 2020; Published: July 28, 2020

\begin{abstract}
A novel method for the determination of sulfamethazine $\left(\mathrm{SM}_{2}\right)$, sulfamonomethoxinc (SMM), sulfamethoxazole (SMZ), sulfadiazine (SD), sulfaquinoxaline (SQX) from the water has been developed using ionic liquid dispersive liquid-liquid microextraction coupled with high performance liquid chromatography. The influence of extraction parameters including types of extraction solvent, volume of extraction solvent, types of dispersant solvent, volume of dispersant solvent, extraction time, and $\mathrm{pH}$ were investigated. Using $80 \mu \mathrm{L}$ 1-Hexyl-3-methylimidazolium hexafluorophosphate $\left(\left[\mathrm{C}_{6} \mathrm{MIM}_{\mathrm{PF}}\right)\right.$ as extraction solvent, $800 \mu \mathrm{L}$ acetonitrile as dispersant solvent, $\mathrm{pH}=4.0$, extraction $3 \mathrm{~min}$, centrifugal after storage $2 \mathrm{~min}$. Under the optima 1 conditions, good linear relationships were obtained in the five sulfonamides residues concentrations of $5 \sim 150 \mu \mathrm{g} \cdot \mathrm{L}^{-1}$ with the correlation coefficients of $0.9988-0.9994$, limits of detection (LOD) were $0.74 \sim 1.21 \mu \mathrm{g} \cdot \mathrm{L}^{-1}(\mathrm{~S} / \mathrm{N}=3)$, limits of quantification (LOQ) were $2.51 \sim 4.06 \mu \mathrm{g} \cdot \mathrm{L}^{-1}(\mathrm{~S} / \mathrm{N}=10)$. The proposed method was applied to the determination of five sulfonamides residues in water, the recoveries were from $88.5 \%$ to $98.8 \%$, with the corresponding intra-day RSDs less than $5.1 \%$, inter-day RSDs less than $9.5 \%$. The method was considered to be simple, fast, and precise to satisfy the requirements of the residual analysis of sulfonamides in water.
\end{abstract}

Keywords: Ionic Liquid, Dispersive Liquid-liquid Microextraction, High Performance Liquid Chromatography, Water

\section{Introduction}

Sulfonamides is a kind of synthetic antibacterial drugs commonly used in animal husbandry, which is widely used in the treatment and prevention of bacterial diseases of livestock and poultry [1] due to its broad spectrum of antibacterial, strong antibacterial ability, rapid absorption and low price. But sulfonamides take longer to metabolize in the body, It is harm to the human body when the concentration exceeds a certain limit. Acute poisoning can be caused by large doses of sulfonamides in a short period of time, long-term small doses of sulfanilamide drugs can cause chronic poisoning, They can damage the body's immune system, urinary system, and tissues. In addition, when sulfonamides are kept in the body for a long time, many fungi in the body will develop resistance to such drugs [2]. Codex alimentarius commission [3], the European Union [4] and China [5] have strictly stipulated the maximum residue limit of $100 \mathrm{~g} \mathrm{~kg}-1$ for the total amount of sulfonamides in animal food. Therefore, how to effectively and quickly monitor sulfanilamide antibiotics in environmental waters is one of the urgent problems in the field of environmental protection

At present, the sulfonamides were detected of high performance liquid chromatography [6, 7] (HPLC), liquid chromatography-tandem mass spectrometry $[8,9]$ (LC-MS/MS) and high performance capillary electrophoresis 
$[10,11]$ (HPCE). HPLC method has been widely used in the analysis of target compounds with high resolution, high sensitivity, good repeatability and low cost. Sample pretreatment is a technique in which the target compounds are extracted, concentrated and purified from the samples before instrumental analysis. The purpose is to make the components to be measured in the sample fully extracted by the extracting agent, and then convert them into a form that can be measured by the analytical instrument, so as to carry out accurate quantitative and qualitative analysis $[12,13]$. It can be seen that sample pretreatment is the most important part of the analysis.

The sample pretreatment methods include solid phase extraction [14], liquid-liquid extraction [15, 16], dispersed solid phase extraction [17, 18], etc. Dispersive liquid-liquid microextraction (DLLME) is a kind of liquid phase extraction technology, the miniaturization in a certain volume of sample solution or sample extract, join the dispersant containing extraction agent, after the oscillation, the extractant and sample solution form emulsion system, enrichment of the target compounds after centrifugal extraction solvent precipitation in the bottom of the sample, suitable for analysis of trace components in water samples. Traditional DLLME often USES volatile and toxic organic reagents as extraction agents, which may do harm to the experimental environment and operators. Ionic liquid is a new type of solvent with environmental protection and excellent performance, which can be used to study a new extraction method for DLLME. This article USES the imidazoles ionic liquid as the extractant, acetonitrile as dispersant, examines the methods on the experimental results, the influence of various factors to establish the DLLME technique combined with HPLC to determine the water residue detection methods of five kinds of sulfa compounds, the low solvent consumption, high extraction efficiency, easy operation, environmentally friendly, for the separation of harmful substances in surface water detection to provide technical support.

\section{Instrumentation and Reagents}

\subsection{Instrumentation}

Chromatographic analysis was performed on e2695 HPLC systems (Waters Co., USA) equipped with a Diode Array Detector. The samples were treated with 5810R centrifuge (Eppendorf Co., Germany), VX200 Vortex oscillator (Labnet Equipment Co., USA). Electronic analytical balance was performed on AR124CN (OHAUS Co., Shanghai), microinjector (Agilent Co., USA), ST5000-F pH meter (OHAUS Co., Shanghai), filter holder, (T50, Tianjin Jinteng Technology Co., Ltd.).

\subsection{Reagents}

\footnotetext{
1-hexyl-3-methylimidazole ([C $\left.{ }_{6} \mathrm{MIM} \mathrm{PF}_{6}\right)$, 1-octyl-3hexafluorophosphate 1-hexyl-3-methylimidazolium
}

hexafluorophosphate methylimidazolium ([OMIM]PF6), trifluoromethylsulfonate
([HMIM]OTF) were obtained from Meryer (Shanghai) Chemical Technology Co., Ltd. Methanol, ethanol and acetonitrile (HPLC grade) were purchased from Fisher Scientific (Fair Lawn, NJ, USA). Sulfamethazine $\left(\mathrm{SM}_{2}\right)$, Sulfamonomethoxinc (SMM), Sulfamethoxazole (SMZ), Sulfadiazine (SD), Sulfaquinoxaline (SQX) of 98\% purity were purchased from the Bellingway Technology Co., Ltd.(Beijing, China). Phosphoric acid, oxalic acid, sodium chloride (AR) were obtained from Macklin Chemical Reagent Co. Ltd. (Shanghai, China). Intubation was obtained from Agilent $(250 \mu \mathrm{L}, \mathrm{USA})$. Canal water, Liming lake water and tap water were collected in the Daqing.

\subsection{Chromatography Conditions}

An Waters e2695 high performance liquid chromatography system with was used for analysis. The separation of the sulfonamides was operated on an Diamonsil $\mathrm{C}_{18}$ column (4.6 $\mathrm{mm} \times 250 \mathrm{~mm}, 5 \mu \mathrm{m})$. The mobile phase was a mix-ture of acetonitrile (A) and $0.1 \%$ formic acid-water (B), and the flow

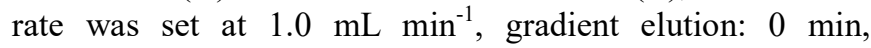
$V_{\mathrm{A}}: V_{\mathrm{B}}=80: 20 ; 5 \mathrm{~min}, V_{\mathrm{A}}: V_{\mathrm{B}}=70: 30 ; 10 \mathrm{~min}, V_{\mathrm{A}}: V_{\mathrm{B}}=60: 40 ; 20$ $\min , V_{\mathrm{A}}: V_{\mathrm{B}}=10: 90$. The column temperature was set at $30{ }^{\circ} \mathrm{C}$, and the chromatographic data was acquired at wavelength for $270 \mathrm{~nm}$. The injection volume was $5 \mu \mathrm{L}$.

\subsection{Experimental Method}

The $5.00 \mathrm{~mL}$ water sample was added into $25 \mathrm{~mL}$ Teflon centrifuge tube, $0.2 \mathrm{~g} \mathrm{NaCl}, 80 \mu \mathrm{L}\left[\mathrm{C}_{6} \mathrm{MIM}^{\mathrm{M}} \mathrm{PF}_{6} 、 800 \mu \mathrm{L}\right.$ acetonitrile were added. The mixed solution of $\mathrm{pH}$ was adjusted between 4 with $0.1 \mathrm{~mol} \mathrm{~L}^{-1}$ of phosphoric acid. After vortex agitating for $2 \mathrm{~min}$, the Teflon was centrifuged for $5 \mathrm{~min}$ at $10000 \mathrm{rpm}$. Finally, $30 \mu \mathrm{L}$ the extraction solution was obtained from bottom of sample solution and transferred into a $250 \mu \mathrm{L}$ sample bottle with intubation for HPLC analysis.

\section{Results and Discussion}

\subsection{Effect of the Type and Volume of Ionic Liquid on the Extraction Efficiency}

In liquid-liquid microextraction, the extractant should have strong solubility in the target compound, but insoluble in water and denser than water, so as to ensure a good extraction efficiency. The effects of $\left[\mathrm{C}_{6} \mathrm{MIM}\right] \mathrm{PF}_{6},[\mathrm{OMIM}] \mathrm{PF}_{6}$ and [HMIM]OTF on the extraction of five sulfonamides in water were investigated. According to the above method, the results showed that $\left[\mathrm{C}_{6} \mathrm{MIM}\right] \mathrm{PF}_{6}$ had better extraction effect on sulfonamide compounds. Therefore, in this experiment, $\left[\mathrm{C}_{6} \mathrm{MIM}\right] \mathrm{PF}_{6}$ was the extraction solvent.

In order to evaluate the effect of the extraction solvent volume on extraction efficiency, a series of experiments was designed based on changing $\left[\mathrm{C}_{6} \mathrm{MIM}\right] \mathrm{PF}_{6}$ volumes within the range from 40 to $120 \mu \mathrm{L}$. Figure 1 shows the effect of extraction solvent volume on extraction recoveries. The largest recoveries were obtained at $80 \mu \mathrm{L}$ of $\left[\mathrm{C}_{6} \mathrm{MIM}\right] \mathrm{PF}_{6}$ for five sulfonamides. Further increase in $\left[\mathrm{C}_{6} \mathrm{MIM}\right] \mathrm{PF}_{6}$ volume 
resulted in a decrease in recoveries. Therefore, $80 \mu \mathrm{L}$ of $\left[\mathrm{C}_{6} \mathrm{MIM}\right] \mathrm{PF}_{6}$ was chosen as the optimal type and volume of extraction solvent in this study.

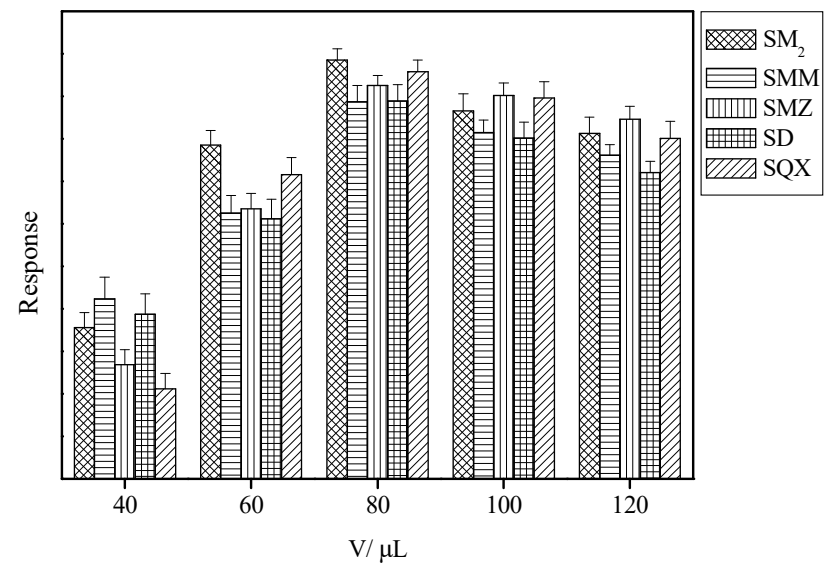

Figure 1. Effect of extractant volume on extraction efficiency.

\subsection{Effect of the Type and Volume of Dispersing Solvent on the Extraction Efficiency}

The type and volume of dispersants are important factors affecting the extraction efficiency. The effects of methanol, acetonitrile and acetone as dispersants on the recovery of sulfonamides were investigated. The dispersing solvent $800 \mu \mathrm{L}$, including $80 \mu \mathrm{L}\left[\mathrm{C}_{6} \mathrm{MIM}\right] \mathrm{PF}_{6}$, was added to $5 \mathrm{~mL}$ water samples with added concentration of $50 \mu \mathrm{g} \cdot \mathrm{L}^{-1}$. The water samples were vortex oscillated for $3 \mathrm{~min}$, centrifuged for $5 \mathrm{~min}$, and the bottom layer liquid was taken for chromatographic analysis. The results showed that the extraction recovery of ethanol and acetone as dispersants was relatively low, and the experimental results were not stable. The response value of sulfonamides was the largest when acetonitrile was used as dispersant, and the recovery results were satisfactory. Therefore, acetonitrile was selected as dispersant in this experiment.

The response value of target compound was increased with increasing volume of acetonitrile between 400 to $1400 \mu \mathrm{L}$. When the volume of acetonitrile was more than $800 \mu \mathrm{L}$, the response value of target compound decreased. Therefore, in subsequent experiments, $800 \mu \mathrm{L}$ of acetonitrile was used as the dispersing solvent.

\subsection{Effect of the Extraction Time on the Extraction Efficiency}

It takes a certain time to establish the equilibrium of liquid-liquid microextraction. The effects of extraction time of
$1,2,3,4$ and $5 \mathrm{~min}$ on the extraction efficiency were investigated. The experimental results showed that the distribution proportion of the target compound in the ionic liquid extractant and water phase reached equilibrium within 2 min. If the extraction time continues to increase, the extraction efficiency of the target compound will not change much, so the ultrasonic vibration time is chosen as $2 \mathrm{~min}$ in the experiment.

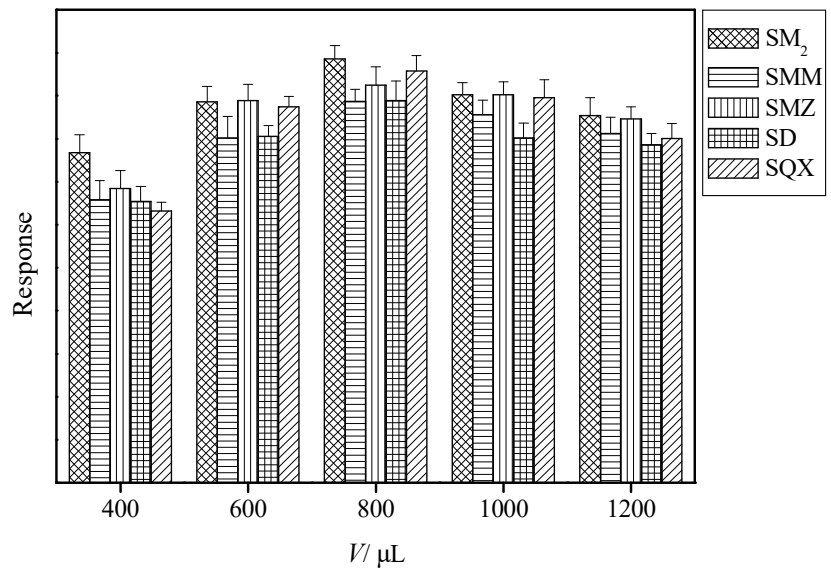

Figure 2. Effect of dispersant volume on extraction efficiency.

\subsection{Effect of the pH on the Extraction Efficiency}

In the extraction process, the $\mathrm{pH}$ value of the solution can affect the extraction efficiency. In this paper, the influence of different $\mathrm{pH}$ values $(3.0,4.0,5.0,6.0,7.0,8.0)$ of the sample solution on the extraction efficiency was investigated. The experimental results showed that when the $\mathrm{pH}$ value was 4.0, The response value of five sulfonamides was the highest. This may be because the dissociation constant of sulfonamides is in the range of 3.7-4.7. When the $\mathrm{pH}$ of the sample solution is 4.0, the solubility of the target compound is the largest, the amount transferred to the extractant species is the largest, and the extraction efficiency is the best, so the $\mathrm{pH}$ of the solution is 4.0.

\section{Evaluation of Methods}

\subsection{Linearity, Lod and Loq}

Under the condition of the best optimization experiment, The mixed standard solution of five sulfonamides compound were added into water samples, the linear correlation coefficient, repeatability, LOD, $(\mathrm{S} / \mathrm{N}=3)$ and LOQ $(\mathrm{S} / \mathrm{N}=10)$ of the establised were evaluated, the results are shown in Table 1.

Table 1. Correlation coefficients, linear ranges, LOD, LOQ, RSD of five kinds of SAs.

\begin{tabular}{llllll}
\hline SAs & $\mathbf{r}$ & linear ranges $/ \boldsymbol{\mu g} \cdot \mathbf{L}^{-1}$ & $\mathbf{L O D} / \boldsymbol{\mu g} \cdot \mathbf{L}^{-1}$ & $\mathbf{L O Q} / \boldsymbol{\mu g} \cdot \mathbf{L}^{-1}$ & $\mathbf{R S D} / \mathbf{\%}$ \\
\hline $\mathrm{SM}_{2}$ & 0.9991 & $5 \sim 500$ & 0.74 & 2.51 & 3.4 \\
$\mathrm{SMM}$ & 0.9995 & $5 \sim 500$ & 0.85 & 2.85 & 4.2 \\
$\mathrm{SMZ}$ & 0.9988 & $10-500$ & 0.94 & 3.25 & 2.6 \\
$\mathrm{SD}$ & 0.9993 & $10-500$ & 0.81 & 3.12 & 3.6 \\
$\mathrm{SQX}$ & 0.9981 & $10-500$ & 1.21 & 4.06 & 4.8 \\
\hline
\end{tabular}


Table 2. Recoveries and RSDs of the five SAs spiked in water at three levels (\%).

\begin{tabular}{|c|c|c|c|c|c|c|c|c|c|}
\hline \multirow{3}{*}{ SAs } & \multicolumn{3}{|c|}{ Spiked level $\left(5 \mu \mathrm{g} \cdot \mathrm{L}^{-1}\right)$} & \multicolumn{3}{|c|}{ Spiked level $\left(10 \mu \mathrm{g} \cdot \mathrm{L}^{-1}\right)$} & \multicolumn{3}{|c|}{ Spiked level $\left(50 \mu \mathrm{g} \cdot \mathrm{L}^{-1}\right)$} \\
\hline & $\begin{array}{l}\text { Average } \\
\text { recovery }\end{array}$ & Intra-day & inter-day & $\begin{array}{l}\text { Average } \\
\text { recovery }\end{array}$ & Intra-day & inter-day & $\begin{array}{l}\text { Average } \\
\text { recovery }\end{array}$ & Intra-day & inter-day \\
\hline & & & RSD & & & RSD & & & RSD \\
\hline SM2 & 89.6 & 3.6 & 6.8 & 94.2 & 2.7 & 7.5 & 96.7 & 4.6 & 9.5 \\
\hline SMM & 90.2 & 4.2 & 7.8 & 90.2 & 3.8 & 9.2 & 98.8 & $3 . .3$ & 7.6 \\
\hline SMZ & 88.5 & 2.4 & 6.4 & 89.6 & 4.0 & 6.6 & 95.2 & 2.6 & 7.9 \\
\hline SD & 93.6 & 4.1 & 8.7 & 88.7 & 3.9 & 8.2 & 89.6 & 5.1 & 9.2 \\
\hline SQX & 88.9 & 4.6 & 9.1 & 93.3 & 2.9 & 8.5 & 95.4 & 3.4 & 7.8 \\
\hline
\end{tabular}

\subsection{Accuracy and Precision}

For the determination of the accuracy, recovery experiments were performed using three different concentration levels $\left(5,10\right.$, and $\left.50 \mu \mathrm{g} \cdot \mathrm{L}^{-1}\right)$ of spiked samples with known amounts of five sulfonamides. The RSD of the standard sample recovery rate was analyzed for 6 times in a day, and the RSD of the standard sample recovery rate was analyzed for 3 days in a row. It can be seen from table 2 that the standard recovery rate of sulfonamides was between 88.5 and $98.8 \%$, and the intra-day precision was lower than $5.1 \%$

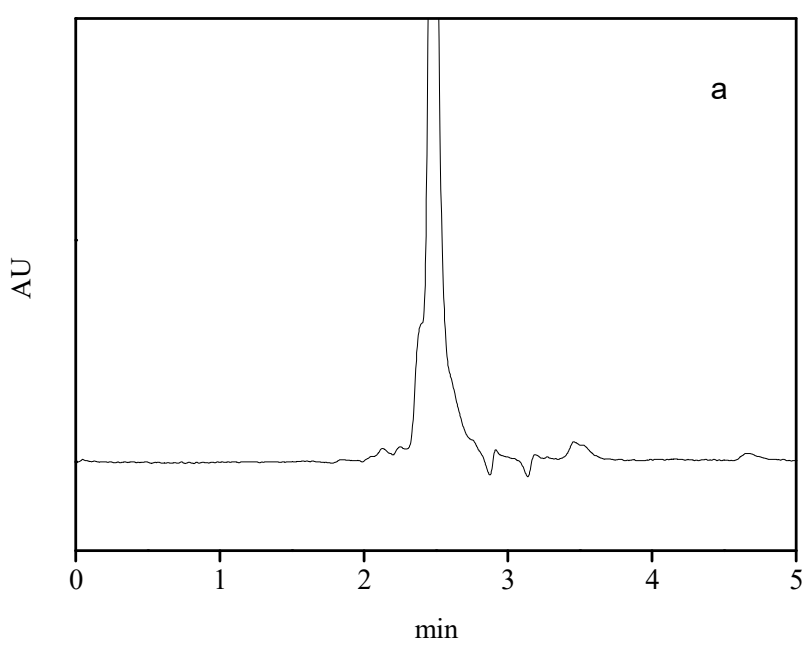

and the intra-day precision was lower than $9.5 \%$.

\subsection{Analysis of Real Sample}

Under the optimum conditions, the samples of canal water, tap water, lake water and standard water were analyzed by ion-liquid-dispersion-liquid-liquid microextraction and high performance liquid chromatography. The results showed that sulfonamides were no detected in tap water, canal water and lake water. Chromatogram of blank sample and standard sample is shown in Figure 3.

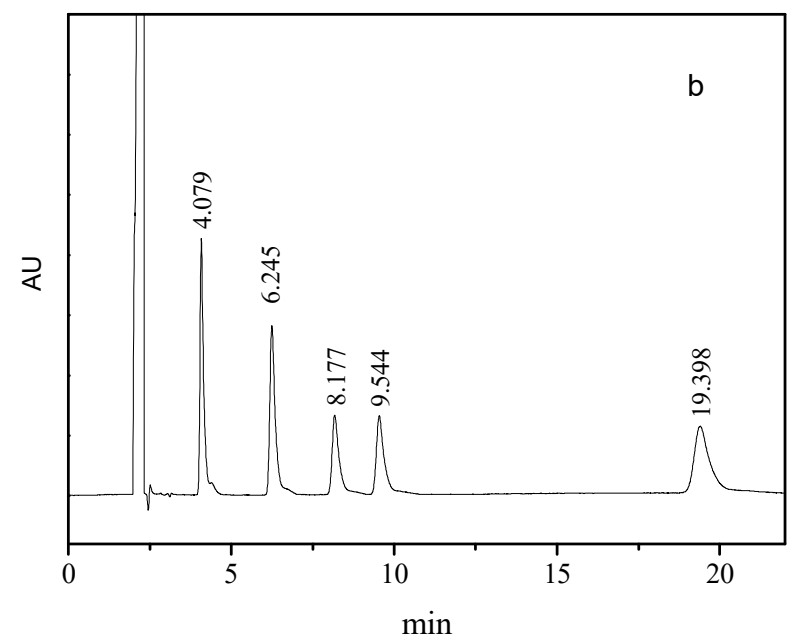

$\left(\mathrm{SM}_{2}, \mathrm{t}_{\mathrm{R}}=4.079 \mathrm{~min} ; \mathrm{SMM}, \mathrm{t}_{\mathrm{R}}=6.245 \mathrm{~min} ; \mathrm{SMZ}, \mathrm{t}_{\mathrm{R}}=8.177 \mathrm{~min} ; \mathrm{SD}, \mathrm{t}_{\mathrm{R}}=9.544 \mathrm{~min}, \mathrm{SQX}, \mathrm{t}_{\mathrm{R}}=19.398 \mathrm{~min}\right)$.

Figure 3. Chromatograms of (a) a blank water sample (b) a spiked sample.

\section{Conclusion}

In this study, a method for the determination of five sulfonamides in water by ionic liquid dispersion liquid-liquid microextraction combined with high performance liquid chromatography (HPLC) was established. Meanwhile, the effects of the type and volume of ionic liquid, the type and volume of dispersant, extraction time and $\mathrm{pH}$ on the extraction efficiency were investigated, and the best extraction conditions were determined. The developed method has the advantages of high extraction efficiency, simplicity, rapidity and reduction of the use of toxic and harmful organic solvents. The recovery experiments of local tap water, canal water and lake water have been carried out, and the satisfactory results have been obtained, which shows that the established method has a good development prospect for the detection of sulfonamides in actual water samples.

\section{Funding}

This work was supported by the Heilongjiang Bayi Agricultural University Support Program (2019-KYYWF-0502， ZRCPY201920， PTJH201905), Heilongjiang Reclamation Area General Bureau Support Program (HKKYZD190717).

\section{Conflicts of Interest}

The authors declare that they have no competing interests.

\section{Ethical Approval}

This article does not contain any studies with human 
participants or animals performed by any of the authors.

\section{Informed Consent}

Informed consent is not applicable to this study.

\section{Acknowledgements}

Heilongjiang Province Cultivating Collaborative Innovation Center for The Beidahuang Modern Agricultural Industry Technology; Heilongjiang Plant Growth Regulator Engineering Technology Research Center.

\section{References}

[1] Yinming Fan, Guolong Zeng, Xiaoguo Ma. Effects of prepolymerization on surface molecularly imprinted polymer for rapid separation and analysis of sulfonamides in water [J]. Journal of Colloid and Interface Science, 2020, 5711: 21-29.

[2] Yeliz Demir, Zeynep Köksal. The inhibition effects of some sulfonamides on human serum paraoxonase-1 (hPON1) [J]. Pharmacological Reports, 2019, 71: 545-549.

[3] Codex Alimentarius Commossion. CAC/MRL 02-2005: 21 [Z].

[4] European Agency for the Evaluation of Medicinal Products. EMEA/MRL/878/03- FINAL, Metamizole summary report [R]. London: The European Agency for the Evaluation of Medicinal Products, 2003.

[5] Announcement No. 235 [Z] of the Ministry of agriculture of the people's Republic of China.

[6] H Sereshti, M Khosraviani, MS Amini-Fazl. Miniaturized salting-out liquid-liquid extraction in a coupled-syringe system combined with HPLC-UV for extraction and determination of sulfanilamide [J]. Talanta, 2014, 121: 199-204.

[7] W Yu, K Li, ZL Liu, et al. Novelty aqueous two-phase extraction system based on ionic liquid for determination of sulfonamides in blood coupled with high-performance liquid chromatography [J]. Microchemical Journal, 2018, 136: 263-269.

[8] Jiangyi Wu, Yanyun Li, Weicheng Li, Zhenbin Gong, Xiaojia Huang. Preparation of a novel monolith-based adsorbent for solid-phase microextraction of sulfonamides in complex samples prior to HPLC-MS/MS analysis [J]. Analytica Chimica Acta, 2020, 111829: 9-17.
[9] Carolina Nebot, Patricia Regal, Jose Manuel Miranda, Cristina Fente, Alberto Cepeda. Rapid method for quantification of nine sulfonamides in bovine milk using HPLC/MS/MS and without using SPE [J]. Food Chemistry, 2013, 141 (31): 2294-2299.

[10] G Font, A Juan-García, Y Picó. Pressurized liquid extraction combined with capillary electrophoresis-mass spectrometry as an improved methodology for the determination of sulfonamide residues in meat [J]. Journal of Chromatography A, 2007, 1159 (1-23): 233-241.

[11] LR Yang, SJ Zhou, YZ Xiao, et al. Sensitive simultaneous determination of three sulfanilamide artificial sweeters by capillary electrophoresis with on-line preconcentration and contactless conductivity detection [J]. Food Chemistry, 2015, 1881: 446-451.

[12] Sun Peng, Qiao Sshi, Guo Xiujuan. Determination of enrofloxacin and ciprofloxacin in liquid milk by using ionic liquid-based dispersive liquid-phase microextraction coupled with HPLC [J]. Chin. J. Pharm. Anal., 2017, 37: 111-116.

[13] Sun Peng, Gao Yuling, Wang Shuang, et al. Determination of enrofloxacin and ciprofloxacin in liquid milk by using ionic liquid-based dispersive liquid-phase microextraction coupled with high performance liquid chromatography $[\mathrm{J}]$ Chemical Reagent, 2016, 38: 999-1002.

[14] AV Herrera-Herrera, J Hernández-Borges, MM Afonso, et al. Comparison between magnetic and non magnetic multi-walled carbon nanotubes-dispersive solid-phase extraction combined with ultra-high performance liquid chromatography for the determination of sulfonamide antibiotics in water samples [J]. Talanta, 2013, 1161: 695-703.

[15] NE Markina, AV Markin, K Weber, et al. Liquid-liquid extraction-assisted SERS-based determination of sulfamethoxazole in spiked human urine [J]. Analytica Chimica Acta, 2020, 1109: 61-68.

[16] LKadziński, RBanasiuk, B Banecki. Determination of ten sulfonamides in honey using tetrahydrofuran Salting Out Liquid Liquid Extraction and monolithic silica column [J]. LWT, 2018, 96: 7-12.

[17] P Sun, YL Gao, YF Lian. Determination of eugenol in aquatic products by dispersive solid-phase extraction and ultra-high-performance liquid chromatography-tandem mass spectrometry, Food Anal. Methods, 2017, 10: 3217-3224.

[18] P Sun, YL Gao, C Xu, et al. Determination of seven pyrethroid pesticides residue in vegetable samples by gas chromatography using carboxylated multi-walled carbon nanotubes as dispersion solid phase extraction sorbent, Food Additives \& Contaminants: Part A, 2017, 34 (12): 2164-2172. 